\title{
Nanoscale Plasmonic Devices Based on Metal-Dielectric-Metal Stub Resonators
}

\author{
Yin Huang, ${ }^{1,2}$ Changjun Min, ${ }^{2}$ Liu Yang, ${ }^{1,2}$ and Georgios Veronis ${ }^{1,2}$ \\ ${ }^{1}$ Department of Electrical and Computer Engineering, Louisiana State University, Baton Rouge, LA 70803, USA \\ ${ }^{2}$ Center for Computation and Technology, Louisiana State University, Baton Rouge, LA 70803, USA
}

Correspondence should be addressed to Georgios Veronis, gveronis@lsu.edu

Received 10 May 2012; Accepted 10 July 2012

Academic Editor: Qiwen Zhan

Copyright (C) 2012 Yin Huang et al. This is an open access article distributed under the Creative Commons Attribution License, which permits unrestricted use, distribution, and reproduction in any medium, provided the original work is properly cited.

\begin{abstract}
We review some of the recent research activities on plasmonic devices based on metal-dielectric-metal (MDM) stub resonators for manipulating light at the nanoscale. We first introduce slow-light subwavelength plasmonic waveguides based on plasmonic analogues of periodically loaded transmission lines and electromagnetically induced transparency. In both cases, the structures consist of a MDM waveguide side-coupled to periodic arrays of MDM stub resonators. We then introduce absorption switches consisting of a MDM plasmonic waveguide side-coupled to a MDM stub resonator filled with an active material.
\end{abstract}

\section{Introduction}

Light-guiding structures which allow subwavelength confinement of the optical mode are important for achieving compact integrated photonic devices. The minimum confinement of a guided optical mode in dielectric waveguides is set by the diffraction limit and is of the order of $\lambda_{0} / n$, where $\lambda_{0}$ is the wavelength in free space and $n$ is the refractive index.

As opposed to dielectric waveguides, plasmonic waveguides, based on surface plasmons propagating at metaldielectric interfaces, have shown the potential to guide and manipulate light at deep subwavelength scales $[1,2]$. Several different nanoscale plasmonic waveguiding structures have been recently proposed, such as metallic nanowires, metallic nanoparticle arrays, $\mathrm{V}$-shaped grooves, and metal-dielectricmetal (MDM) waveguides [3-10]. Among these, MDM plasmonic waveguides are of particular interest because they support modes with deep subwavelength scale and high group velocity over a very wide range of frequencies extending from DC to visible [11]. Thus, MDM waveguides could be potentially important in providing an interface between conventional optics and subwavelength electronic and optoelectronic devices. Because of the predicted attractive properties of MDM waveguides, their modal structure has been studied in great detail $[6,11-14]$, and people have also started to explore such structures experimentally [1517]. Recent research work has therefore focused on the development of functional plasmonic devices, including active devices, for nanoscale plasmonic integrated circuits. Threedimensional MDM plasmonic waveguides were recently experimentally realized and characterized by several research groups [18-21]. In these experiments, the MDM waveguides are typically defined using electron beam lithography (EBL) and patterned using a focused ion beam (FIB) or other similar processes. In addition, the integration of nanoplasmonic waveguides with active materials, such as photochromic molecules or CdSe quantum dots, has also been recently achieved experimentally by several research groups $[22,23]$.

Waveguide-cavity systems are particularly useful for the development of several integrated photonic devices, such as tunable filters, optical switches, channel drop filters, reflectors, and impedance matching elements. In MDM plasmonic waveguide devices, a waveguide-cavity system can be created by side-coupling a stub resonator, consisting of a MDM waveguide of finite length, to a MDM waveguide [9]. In this paper, we provide a review of some of our own recent research activities on plasmonic devices based on metaldielectric-metal stub resonators for manipulating light at the nanoscale [24-26]. The remainder of the paper is organized as follows. In Section 2, we first review the methods used 
for the simulation and analysis of such devices. We then introduce slow-light waveguides for enhanced light-matter interaction (Section 3) and absorption switches (Section 4) based on metal-dielectric-metal stub resonators. Finally, our conclusions are summarized in Section 5.

\section{Simulation and Analysis Methods}

2.1. Full-Wave Finite-Difference Methods. The properties of MDM plasmonic waveguide devices can be investigated using full-wave electromagnetic simulation methods such as finite-difference methods in the time and frequency domains. In particular, the finite-difference frequencydomain (FDFD) method $[27,28]$ allows to directly use experimental data for the frequency-dependent dielectric constant of metals such as silver [29], including both the real and imaginary parts, with no approximation. Perfectly matched layer (PML) absorbing boundary conditions are used at all boundaries of the simulation domain [30].

Due to the rapid field variation at the metal-dielectric interfaces, a very fine grid resolution of $\sim 1 \mathrm{~nm}$ is required at the metal-dielectric interfaces to adequately resolve the local fields. On the other hand, a grid resolution of $\sim \lambda / 20$ is sufficient in other regions of the simulation domain. For example, the required grid size in air at $\lambda_{0}=1.55 \mu \mathrm{m}$ is $\sim 77.5 \mathrm{~nm}$, which is almost two orders of magnitude larger than the required grid size at the metal-dielectric interfaces. A nonuniform orthogonal grid [31] is therefore commonly used to avoid an unnecessary computational cost. We found that by using such a grid our results are accurate to $\sim 0.05 \%$.

The properties of MDM plasmonic waveguide devices can also be investigated using the finite-element frequencydomain method (FEM). The FEM is a more powerful technique than FDFD, especially for problems with complex geometries. However, FDFD is conceptually simpler and easier to program. The main advantage of FEM is that complex geometric structures can be discretized using a variety of elements of different shapes, while in FDFD a rectangular grid is typically used leading to staircase approximations of particle shapes [30, 32]. In addition, in FEM fields within elements are approximated by shape functions, typically polynomials, while in FDFD a simpler piecewise constant approximation is used [32]. In short, FEM is more complicated than FDFD but achieves better accuracy for a given computational cost [32].

2.2. Transmission Line Theory. The properties of systems, which consist of circuits of deep subwavelength MDM plasmonic waveguides, can be described using the concept of characteristic impedance and transmission line theory $[7,33,34]$.

The characteristic impedance of the fundamental TEM mode in a perfect electric conductor (PEC) parallel-plate waveguide with a dielectric layer thickness $d$ is uniquely defined as the ratio of voltage $V$ to surface current density $I$ and is equal to [34]

$$
Z_{\mathrm{TEM}} \equiv \frac{V}{I}=\frac{E_{x} w}{H_{y}}=\frac{\beta_{\mathrm{TEM}}}{\omega \varepsilon_{0}} w=\sqrt{\frac{\mu_{0}}{\varepsilon_{0}}} w
$$

where $E_{x}, H_{y}$ are the transverse components of the electric and magnetic field, respectively, and we assumed a unitlength waveguide in the $y$ direction. Non-TEM modes, such as the fundamental MDM mode, voltage, and current, are not uniquely defined. However, metals like silver satisfy the condition $\left|\varepsilon_{\text {metal }}\right| \gg \varepsilon_{\text {diel }}$ at the optical communication wavelength of $1.55 \mu \mathrm{m}$ [29]. Thus, $\left|E_{x \text { metal }}\right| \ll\left|E_{x \text { diel }}\right|$, so that the integral of the electric field in the transverse direction can be approximated by $E_{x \operatorname{diel}} w$, and we may therefore define the characteristic impedance of the fundamental MDM mode as

$$
Z_{\mathrm{MDM}}(w) \equiv \frac{E_{x \operatorname{diel}} w}{H_{y \text { diel }}}=\frac{\gamma_{\mathrm{MDM}}(w)}{j \omega \varepsilon} w,
$$

where $\gamma_{\mathrm{MDM}}=\alpha_{\mathrm{MDM}}+i \beta_{\mathrm{MDM}}$ is the complex wave vector of the fundamental propagating TM mode in a MDM waveguide of width $w$ and $\varepsilon$ is the dielectric permittivity of the dielectric region of the MDM waveguide.

2.3. Scattering Matrix Theory. The properties of systems which consist of circuits of deep subwavelength MDM plasmonic waveguides, in which only the fundamental TM mode is propagating, can also be described using scattering matrix theory [35]. As mentioned above, in the transmission line theory method the transmission and reflection coefficients at MDM waveguide junctions are calculated using the concept of the characteristic impedance (2). Unlike the transmission line theory method, in the scattering matrix theory method, the transmission and reflection coefficients at MDM waveguide junctions are directly numerically extracted using a fullwave simulation method such as FDFD [35]. Thus, the use of the scattering matrix theory method results in improved accuracy and increased computational cost associated with the additional full-wave simulations required to extract the transmission and reflection coefficients at MDM waveguide junctions.

2.4. Numerical Example. We now consider a specific numerical example in order to compare the different methods for the analysis and simulation of nanoscale plasmonic devices based on metal-dielectric-metal stub resonators. We consider a plasmonic MDM waveguide side-coupled to two MDM stub resonators (Figure 1(a)). The resonant frequencies of the cavities can be tuned by adjusting the cavity lengths $L_{1}$ and $L_{2}$. This system is a plasmonic analogue of electromagnetically induced transparency (EIT) [36, 37].

The MDM waveguide and MDM stub resonators have deep subwavelength widths $(w \ll \lambda)$, so that only the fundamental TM mode is propagating. Thus, we can use transmission line theory or scattering matrix theory to account for the behavior of the system. First, the properties of such a side-coupled-cavity structure can be described using transmission line theory and the concept of characteristic impedance. Based on transmission line theory, the structure of Figure 1(a) is equivalent to two short-circuited transmission line resonators of lengths $L_{1}$ and $L_{2}$, propagation constant $\gamma_{\mathrm{MDM}}(w)$, and characteristic impedance $Z_{\mathrm{MDM}}(w)$ (2) which are connected in series to a transmission line with the same characteristic impedance $Z_{\mathrm{MDM}}(w)$ [33]. Based on 


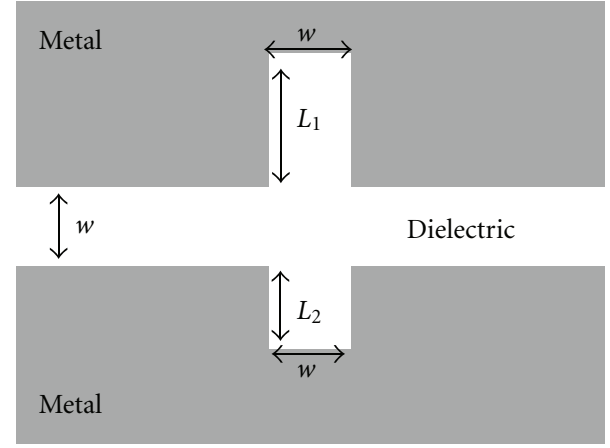

(a)

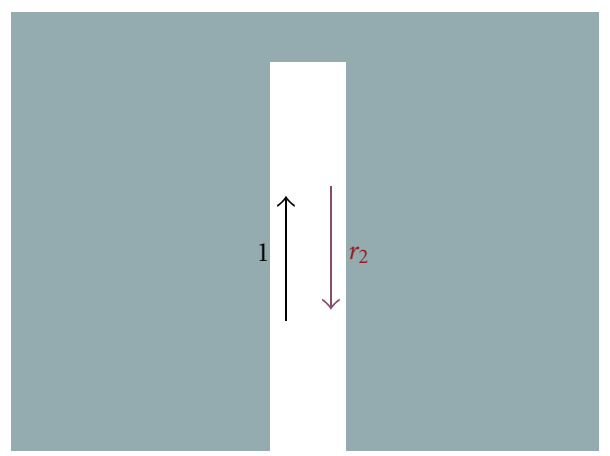

(c)

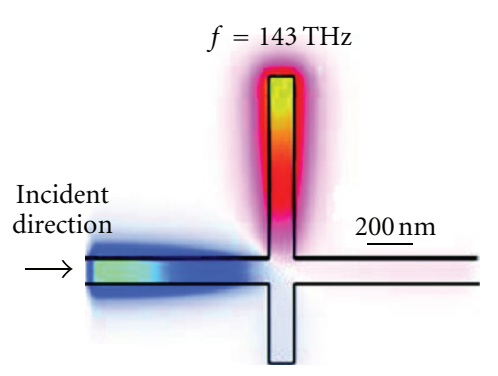

(e)
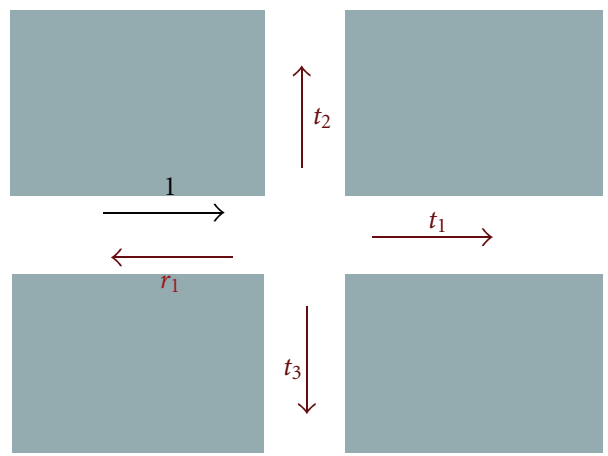

(b)

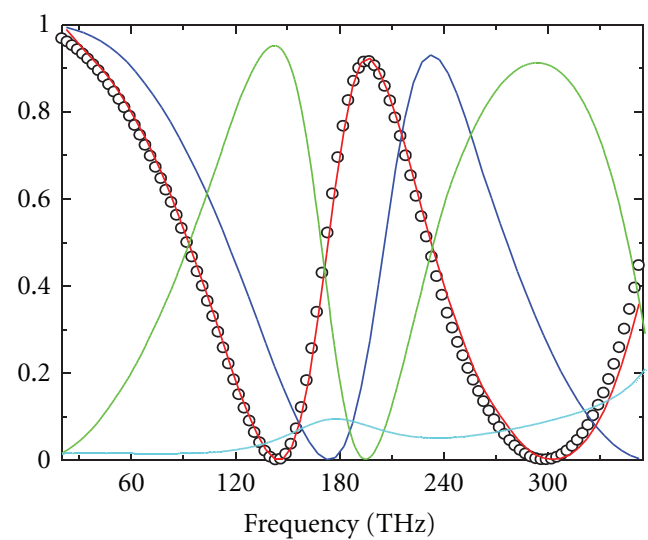

(d)

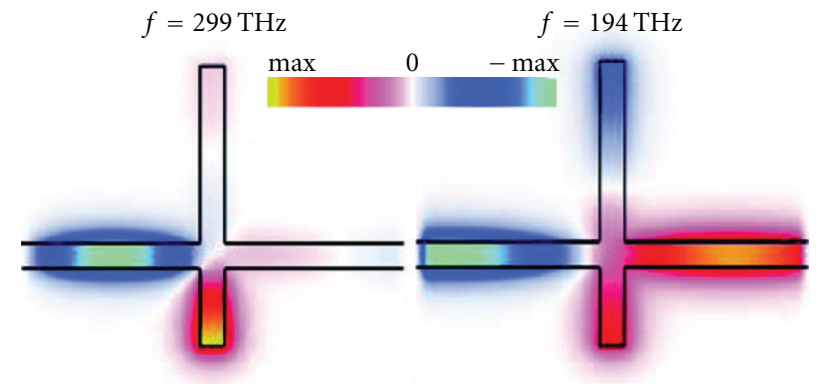

(f)

(g)

Figure 1: (a) Schematic of a MDM plasmonic waveguide side-coupled to two MDM stub resonators. (b) Schematic defining the reflection coefficient $r_{1}$ and transmission coefficients $t_{1}, t_{2}, t_{3}$ when the fundamental TM mode of the MDM waveguide is incident at a waveguide crossing. Note that $t_{2}=t_{3}$ due to symmetry. (c) Schematic defining the reflection coefficient $r_{2}$ of the fundamental TM mode of the MDM waveguide at the boundary of a short-circuited MDM waveguide. (d) Transmission spectra for the structure of (a) calculated using FDFD (circles), transmission line theory (solid blue line), and scattering matrix theory (solid red line) for a silver-air structure with $w=50 \mathrm{~nm}$. Also shown are the reflection (solid green line) and absorption (solid cyan line) spectra calculated using FDFD. Results are shown for $L_{1}=360 \mathrm{~nm}, L_{2}=160 \mathrm{~nm}$. ((e)-(g)) Magnetic field profiles for the structure of (a) for $L_{1}=360 \mathrm{~nm}, L_{2}=160 \mathrm{~nm}, w=50 \mathrm{~nm}$ at $f=143$, 299, $194 \mathrm{THz}$, when the fundamental TM mode of the MDM waveguide is incident from the left.

this model, the transmission $T$ of the structure of Figure 1(a) can be calculated as [33]

$$
T=\left|1+\frac{1}{2}\left(\tanh \left(\gamma_{\mathrm{MDM}} L_{1}\right)+\tanh \left(\gamma_{\mathrm{MDM}} L_{2}\right)\right)\right|^{-2} \text {. }
$$

Second, the properties of such a side-coupled-cavity structure can also be described using scattering matrix theory. The complex magnetic field reflection coefficient $r_{1}$ and transmission coefficients $t_{1}, t_{2}=t_{3}$ for the fundamental propagating
TM mode at a MDM waveguide crossing (Figure 1(b)), as well as the reflection coefficient $r_{2}$ at the boundary of a short-circuited MDM waveguide (Figure 1(c)), are numerically extracted using FDFD [35]. The power transmission spectra $T(\omega)$ of the two-cavity system (Figure $1(\mathrm{a})$ ) can then be calculated using scattering matrix theory as

$$
T=\left|t_{1}-C\right|^{2}
$$


Here, $C=t_{2}^{2}\left(2 t_{1}-2 r_{1}+s_{1}+s_{2}\right) /\left(t_{1}^{2}-\left(r_{1}-s_{1}\right)\left(r_{1}-s_{2}\right)\right)$, $s_{i}=r_{2}^{-1} \exp \left(2 \gamma_{\mathrm{MDM}} L_{i}\right), i=1,2$.

In Figure 1(d) we show the transmission spectra for the structure of Figure 1(a) calculated using FDFD, transmission line theory (3) and scattering matrix theory (4). We observe that there is good agreement between the transmission line theory results and the exact results obtained using FDFD. We note, however, that while the transmission at the transparency peak is correctly predicted by transmission line theory, the resonance frequency obtained using transmission line theory is blue-shifted with respect to the exact result obtained using FDFD (Figure 1(d)). The difference between the transmission line theory results and the exact results obtained using FDFD is due to the error introduced by the transmission line model in the phase of the reflection coefficient $[38,39]$ at the interfaces of the two-side-coupled cavities. Such limitations of the transmission line model for circuits of MDM plasmonic waveguides are also described in detail in [35]. We also observe that there is excellent agreement between the scattering matrix theory results and the exact results obtained using FDFD. This is due to the fact that, as mentioned above, in the scattering matrix theory method, the transmission and reflection coefficients are directly extracted using FDFD. The improved accuracy of scattering matrix theory with respect to transmission line theory comes at the cost of the additional FDFD simulations required to numerically extract the transmission and reflection coefficients.

The transmission spectra $T(\omega)$ feature two dips (Figure $1(\mathrm{~d}))$. We found that the frequencies $\omega_{1}, \omega_{2}$ where these dips occur are approximately equal to the first resonant frequencies of the two cavities; that is, $\phi_{r_{1}}\left(\omega_{i}\right)+\phi_{r_{2}}\left(\omega_{i}\right)-2 \beta_{\mathrm{MDM}}\left(\omega_{i}\right) L_{i} \approx-2 \pi, i=1,2$, where $\phi_{r_{i}}=\arg \left(r_{i}\right), i=1,2$. When either one of the cavities is resonant, the field intensity in that cavity is high, while the field intensity in the other cavity is almost zero, since it is far from resonance (Figures 1(e) and 1(f)). In addition, the transmission is almost zero, since the incoming wave interferes destructively with the decaying amplitude into the forward direction of the resonant cavity field. The transmission spectra $T(\omega)$ also feature a transparency peak centered at frequency $\omega_{0}$. We found that $\omega_{0}$ is approximately equal to the first resonant frequency of the composite cavity of length $L_{1}+L_{2}+w$ formed by the two cavities; that is, $2 \phi_{r_{2}}\left(\omega_{0}\right)-2 \beta_{\mathrm{MDM}}\left(\omega_{0}\right)\left(L_{1}+L_{2}+w\right) \approx-2 \pi$. Thus, the transmission peak frequency $\omega_{0}$ is tunable through the cavity lengths $L_{1}, L_{2}$. When $\omega=\omega_{0}$, the field intensity is high in the entire composite cavity (Figure $1(\mathrm{~g})$ ), and the transmission spectra exhibit a peak due to resonant tunneling of the incoming wave through the composite cavity. In Figure 1(d) we also show the reflection and absorption spectra for the structure of Figure 1(a). We note that in all cases considered in this paper the length of the MDM stub resonators is much smaller than the propagation length of the supported optical mode in the stubs. Thus, the absorption in the MDM stub resonators is small. This applied to both the two-stub structures considered in this Section, as well as the single-stub structures considered in Section 4 below.

\section{Slow-Light Waveguides}

Slowing down light in plasmonic waveguides leads to enhanced light-matter interaction and could therefore enhance the performance of nanoscale plasmonic devices such as switches and sensors [40-45]. However, in conventional MDM plasmonic waveguides, once the operating wavelength and modal size are fixed, the group velocity of light is not tunable.

3.1. Slow-Light Based on a Plasmonic Analog of Periodically Loaded Transmission Lines. In this section, we first introduce a plasmonic waveguide system, which supports a subwavelength broadband slow-light-guided mode with a tunable slow-down factor at a given wavelength. The structure is a plasmonic analog of the periodically loaded transmission lines used in microwave engineering [33]. Such slow-light plasmonic waveguide systems could be potentially used in nonlinear, switching, and sensing applications.

The structure consists of a MDM waveguide side-coupled to a periodic array of MDM stub resonators (Figure 2(a)). Both the MDM waveguide and MDM stub resonators have deep subwavelength widths $\left(w_{0}, w \ll \lambda\right)$. The periodicity $d$ is also subwavelength $(d \ll \lambda)$, so that the operating wavelength is far from the Bragg wavelength of the waveguide [46] $\left(\lambda \gg \lambda_{\text {Bragg }}\right)$. In addition, the distance between adjacent side-coupled cavities $d-w$ is chosen large enough so that direct coupling between the cavities has a negligible effect on the dispersion relation of the system. This sets a lower limit on the periodicity $d_{\min }$ of the plasmonic waveguide structure. For $w=50 \mathrm{~nm}$ we found that $d_{\min } \sim 80 \mathrm{~nm}$.

Using transmission line theory [33], the dispersion relation between $\omega$ and the Bloch wave vector $\gamma=\alpha+i \beta$ of the entire system is found to be

$$
\begin{aligned}
\cosh (\gamma d)= & \cosh ^{2}\left(\gamma_{0} \frac{d}{2}\right)+\sinh ^{2}\left(\gamma_{0} \frac{d}{2}\right) \\
& +\frac{Z_{1}}{Z_{0}} \sinh \left(\gamma_{0} \frac{d}{2}\right) \cosh \left(\gamma_{0} \frac{d}{2}\right) \tanh \left(\gamma_{1} L\right) .
\end{aligned}
$$

In Figure 2(b), we show the dispersion relation for the plasmonic waveguiding structure of Figure 2(a) calculated using FDFD, which, similar to surface plasmons propagating at a single metal-dielectric interface [46], exhibits a resonance. In the lossless metal case, the resonance frequency $\omega_{\text {res }}$ is the cut-off frequency of the fundamental mode, and for $\omega>\omega_{\text {res }}$, the system has a band gap, supporting a nonpropagating mode with $\beta=0$. In addition, we have $\gamma_{0}=i \beta_{0}, \gamma_{1}=i \beta_{1}$, and $\beta\left(\omega_{\text {res }}\right)=\pi / d$ at the band edge. Using these and (5), we find that the resonance frequency $\omega_{\text {res }}$ is a solution of the following equation:

$$
Z_{1} \tan \left[\beta_{1}\left(\omega_{\text {res }}\right) L\right]=2 Z_{0} \cot \left[\beta_{0}\left(\omega_{\text {res }}\right) \frac{d}{2}\right] .
$$

Thus, unlike in conventional MDM waveguides where $\omega_{\text {res }}$ is equal to the surface plasmon frequency of the metaldielectric interface $\left(\omega_{\text {res }}=\omega_{\text {sp }}\right)$ and is fixed for a given metal [46], in such a plasmonic waveguide system the resonance frequency $\omega_{\text {res }}$ is tunable through its geometric parameters. 


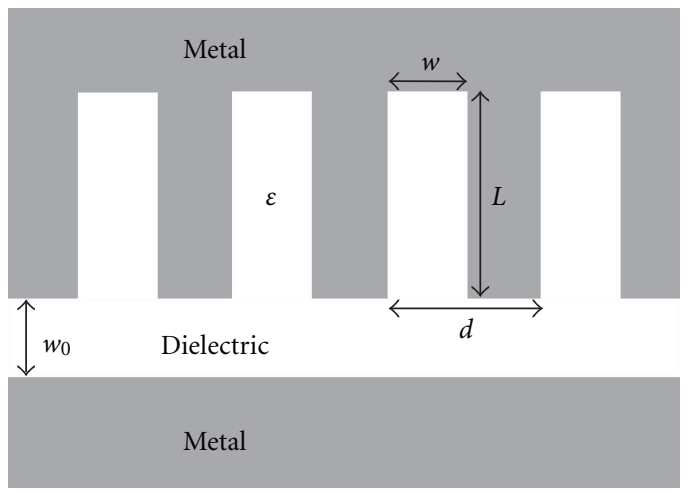

(a)

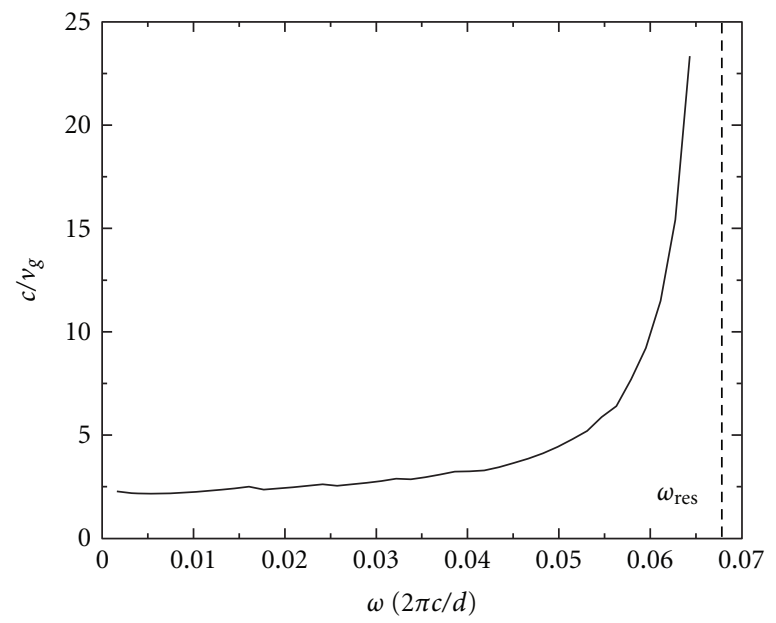

(c)

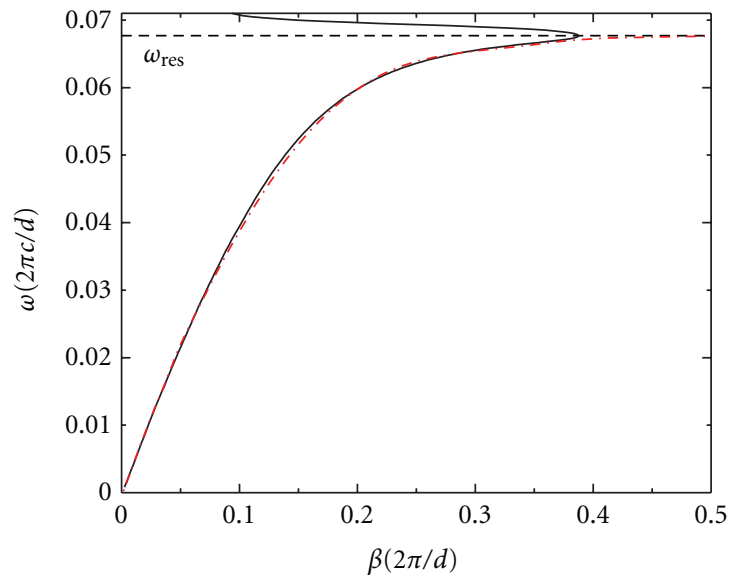

(b)
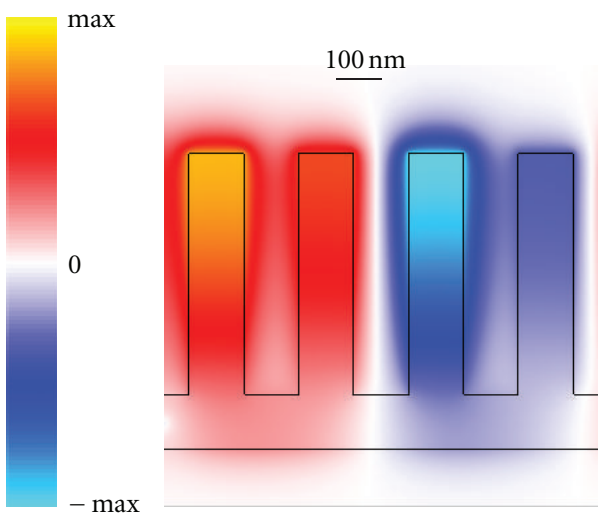

(d)

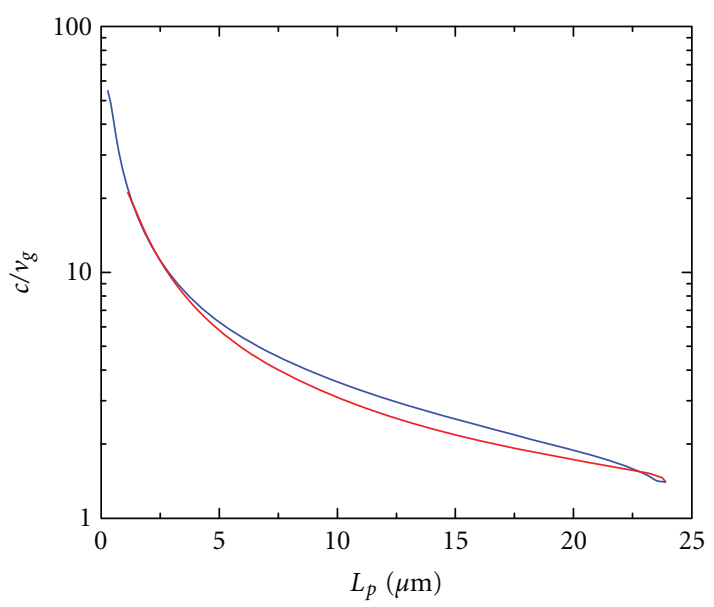

(e)

FIGURE 2: (a) Schematic of a plasmonic waveguide system consisting of a metal-dielectric-metal (MDM) waveguide side-coupled to a periodic array of MDM stub resonators. (b) Dispersion relation of the plasmonic waveguide system of (a) calculated using FDFD (black solid line). Results are shown for a silver-air structure with $d=100 \mathrm{~nm}, L=220 \mathrm{~nm}, w_{0}=w=50 \mathrm{~nm}$. Also shown is the dispersion relation for lossless metal (red dash-dotted line) and the resonance frequency $\omega_{\text {res }}$ (black dashed line) $\left(\omega_{\text {res }} \cong 0.067 * 2 \pi c / d\right.$ corresponding to $\lambda_{\text {res }} \cong 1.5 \mu \mathrm{m}$ ). (c) Reciprocal of the group velocity $v_{g}$ of light in the plasmonic waveguide system as a function of frequency. All parameters are as in (b). (d) Magnetic field profile of the supported optical mode in the system at $\lambda_{0}=1.55 \mu \mathrm{m}$. All other parameters are as in Figure 2(b). (e) Reciprocal of $v_{g}$ versus propagation length $L_{p}$ for the plasmonic waveguide system of Figure 2 (a) at $\lambda_{0}=1.55 \mu \mathrm{m}$ calculated using FDFD. Results are shown for $d=100 \mathrm{~nm}$ (upper blue curve) and $d=200 \mathrm{~nm}$ (lower red curve) as $L$ is varied. All other parameters are as in (b). 
In the presence of loss, we have $\beta\left(\omega_{\text {res }}\right)<\pi / d$ (Figure $2(\mathrm{~b})$ ). In addition, for $\omega>\omega_{\text {res }}$, the Bloch wave vector $\gamma$ has an imaginary component $(\beta \neq 0)$ and the dispersion relation experiences back-bending [46] with negative group velocity $v_{g}=\partial \omega / \partial \beta$ (Figure 2(b)).

In such a plasmonic waveguide system, light is slowed down over a very wide frequency range extending from DC to slightly below the resonance frequency (Figure 2(c)). To find the slow-down factor $c / v_{g}$ in the low-frequency limit, we take the limit of the dispersion relation (5) as $\omega \rightarrow 0$. We note that in the limit of $\omega \rightarrow 0, \gamma_{0} \simeq \gamma_{1} \simeq i \omega \sqrt{\varepsilon \mu_{0}}$. Using these, we obtain the low-frequency $(\omega \rightarrow 0)$ slow-down factor $c / v_{g}=\sqrt{1+w L / w_{0} d}$. We confirmed that this analytical result is in excellent agreement with the result obtained using FDFD. Thus, the group velocity of the system in the low-frequency regime is entirely controlled by its geometry. When $\omega$ approaches $\omega_{\text {res }}\left(\omega \leq \omega_{\text {res }}\right)$, the dispersion relation becomes flat, and the group velocity $v_{g}$ rapidly decreases (Figure 2(c)).

We found that at frequencies far from the resonance frequency, the modal energy of the periodic plasmonic waveguide extends over both the waveguide and the stub resonators. On the other hand, at frequencies near the resonance frequency, the field intensity in the resonators is enhanced, and the modal energy is therefore mostly concentrated in the resonators (Figure $2(\mathrm{~d})$ ). In both cases the modal size is subwavelength. In addition, due to the absorption loss in the metal, there is a trade-off between the slow-down factor $c / v_{g}$ and the propagation length $L_{p}$ of the supported optical mode in such slow-light plasmonic waveguide systems (Figure 2(e)).

3.2. Slow-Light Based on a Plasmonic Analog of Electromagnetically Induced Transparency. In this section, we introduce an alternative MDM plasmonic waveguide system, based on a plasmonic analogue of EIT, which also supports a guided subwavelength slow-light mode. EIT is a coherent process observed in three-level atomic media, which allows a narrow transparency window in the spectrum of an otherwise opaque medium, and can slow down light pulses by several orders of magnitude [47]. Since the EIT spectrum results from the interference of resonant pathways [47, 48], it has been recognized that similar interference effects can also occur in classical systems, such as optical waveguides coupled to resonators and metamaterials [47, 49-51]. In addition, it has been demonstrated that periodic optical waveguides, resulting from cascading structures with EIT-like response, can slow down and even stop light $[48,52,53]$. Our proposed structure consists of a periodic array of two MDM stub resonators side-coupled to a MDM waveguide.

We consider the plasmonic waveguide system (Figure 3(a)) obtained by periodically cascading the sidecoupled-cavity structure of Figure 1(a). The periodicity $d$ is subwavelength $(d \ll \lambda)$, so that the operating wavelength is far from the Bragg wavelength of the waveguide [25] $\left(\lambda \gg \lambda_{\text {Bragg }}\right)$. In addition, the distance between adjacent side-coupled cavities $d-w$ is chosen large enough so that direct coupling between the cavities has a negligible effect on the dispersion relation of the system [25]. Using single-mode scattering matrix theory [35], the dispersion relation between the frequency $\omega$ and the Bloch wave vector $\gamma=\alpha+j \beta$ of the entire system is found to be

$$
\begin{aligned}
\cosh (\gamma d)= & \frac{A}{2} \exp \left[-\gamma_{\mathrm{MDM}}(d-w)\right] \\
& +\frac{B}{2} \exp \left[\gamma_{\mathrm{MDM}}(d-w)\right]
\end{aligned}
$$

which is in excellent agreement with the exact results obtained using FDFD (Figure 4(a)). Here $A=\left(t_{1}-r_{1}\right)\left(\left(t_{1}+\right.\right.$ $\left.\left.r_{1}-2 C\right) /\left(t_{1}-C\right)\right)$ and $B=\left(t_{1}-C\right)^{-1}$. In Figure 3(b), we show the dispersion relation for the plasmonic waveguiding structure of Figure 3(a). In the lossless metal case, the system supports three photonic bands in the vicinity of the cavity resonances. The middle band corresponds to a mode with slow group velocity $v_{g}=\partial \omega / \partial \beta$ and zero group velocity dispersion $\beta_{2}=\partial^{2} \beta / \partial \omega^{2}$ near the middle of this band (Figure 3(b)). In the two band gaps between the three bands, the system supports nonpropagating modes with $\beta=0$. Such a band diagram is similar to that of EIT systems [52]. When losses in the metal are included, the band structure is unaffected in the frequency range of the three bands except at the band edges (Figure 3(b)). In addition, in the frequency range of the two band gaps, the Bloch wave vector $\gamma$ has an imaginary component $(\beta \neq 0)$ and the dispersion relation experiences back-bending [25] with negative group velocity. In Figure 3(c) we show the magnetic field profile for the plasmonic waveguide system of Figure 3(a).

In addition, the width of the middle band and the slowdown factor $c / v_{g}$ strongly depend on the frequency spacing between the resonances $\delta \omega=\omega_{2}-\omega_{1}$. By decreasing the stub lengths difference $\delta L, \delta \omega$ decreases, and this leads to decreased bandwidth of the middle band (Figure $4(\mathrm{a})$ ). In Figures 4(b) and 4(c) we show the slow-down factor $c / v_{g}$ and propagation length $L_{p}$ for the plasmonic waveguide system of Figure 3(a) as a function of frequency for two different values of $\delta L$. In both cases we show the frequency range corresponding to the middle band of the system. For a given $\delta L$, the propagation length $L_{p}$ of the supported optical mode is maximized at a frequency very close to the frequency where the group velocity dispersion is zero. As $\delta L$ and therefore $\delta \omega$ decrease, the slow-down factor $c / v_{g}$ increases, while the propagation length $L_{p}$ decreases at the frequency of zero group velocity dispersion. Thus, there is a trade-off between the slow-down factor $c / v_{g}$ and the propagation length $L_{p}$ of the supported optical mode in such slow-light plasmonic waveguide systems [25]. For $\delta L=200 \mathrm{~nm}(\delta L=75 \mathrm{~nm})$ we have $c / v_{g} \approx 6\left(c / v_{g} \approx 30\right)$ at the frequency where the group velocity dispersion is zero (Figures $4(\mathrm{~b})$ and $4(\mathrm{c})$ ). We found that even larger slow-down factors can be obtained by further decreasing $\delta L$ at the cost of reduced propagation length. We also note that the propagation length of the system for a given slowdown factor can be increased by incorporating gain media in the structure [46]. The slow-down factor exhibits two maxima near the two edges of this band and a minimum at a frequency near the middle of the band where the group velocity dispersion is zero. 


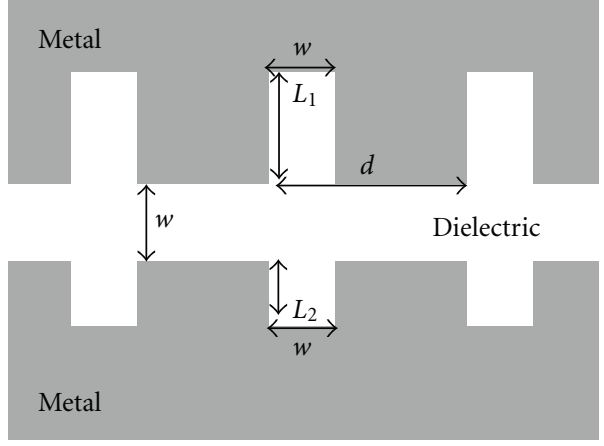

(a)

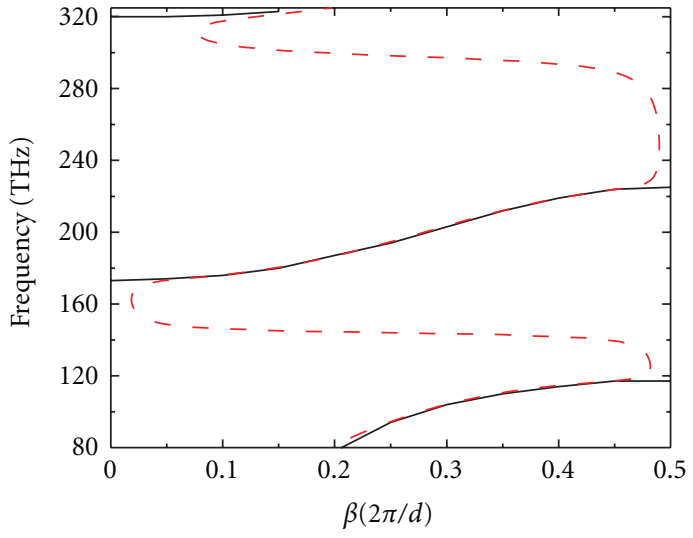

(b)

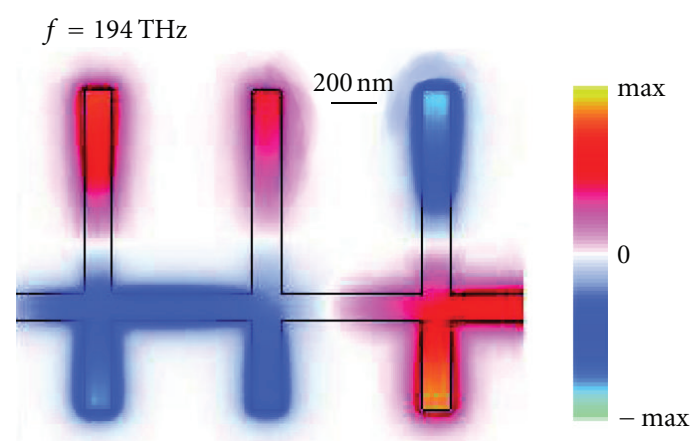

(c)

Figure 3: (a) Schematic of a plasmonic waveguide system consisting of a periodic array of two MDM stub resonators side-coupled to a MDM waveguide. (b) Dispersion relation of the plasmonic waveguide system of Figure 2(a) calculated using FDFD (red dashed line). Results are shown for a silver-air structure with $d=300 \mathrm{~nm}, L_{1}=360 \mathrm{~nm}, L_{2}=160 \mathrm{~nm}$, and $w=50 \mathrm{~nm}$. Also shown is the dispersion relation for lossless metal (black solid line). (c) Magnetic field profile for the structure of (a) for $L_{1}=360 \mathrm{~nm}, L_{2}=160 \mathrm{~nm}, w=50 \mathrm{~nm}$ at $f=194 \mathrm{THz}$.

We also consider the effect of the periodicity $d$ (Figure 3(a)) on the dispersion relation of the system (Figure $4(\mathrm{~d})$ ). For large $d$ the distance $d-w$ between adjacent two-cavity structures in the periodic waveguide is large, so that their coupling through the MDM waveguide is weak. In this regime, the frequency range of the middle band of the periodic waveguide system of Figure 3(a) approximately corresponds to the frequency range of the transparency peak of the two-cavity structure of Figure 1(a). As $d$ decreases, the coupling between adjacent two-cavity structures increases. As a result, the slow-light middle band shifts to higher frequencies, while its width slightly broadens (Figure 4(d)). Thus, the periodicity provides us an additional degree of freedom to tune the dispersion relation of the periodic waveguide system.

\section{Absorption Switches}

One of the main challenges in plasmonics is achieving active control of optical signals in nanoscale plasmonic devices [2]. This challenge has motivated significant recent activities in exploring actively controlled plasmonic devices, such as switches and modulators [22, 23, 54-63]. Several different approaches have been proposed in order to achieve active control of light in nanoscale plasmonic devices [22, 23, 54-63]. These include thermally induced changes in the refractive index [54-56], direct ultrafast optical excitation of the metal [57], as well as the incorporation of nonlinear $[58,59]$, electrooptic $[60,61]$, and gain [62] media in plasmonic devices. An alternative approach for active control of optical signals in plasmonic devices is tuning the absorption coefficient. This has been recently achieved experimentally through optical excitation of photochromic molecules [23] or CdSe quantum dots (QDs) [22, 63].

Here, we consider a switch consisting of a silver-air-silver MDM plasmonic waveguide side-coupled to a MDM stub resonator filled with a material with tunable absorption coefficient $(n=2.02+i \kappa)$ (Figure $5(\mathrm{a}))$. The properties of such a side-coupled-cavity switch can be described using transmission line theory and the concept of characteristic impedance. Based on transmission line theory, the side-coupled-cavity switch is equivalent to a short-circuited transmission line resonator of length $L$, propagation constant $\gamma_{2}$, and characteristic impedance $Z_{2}$, which is connected in series to a transmission line with characteristic impedance $Z_{1}$ [33]. Based on this model, the transmission $T$ of the side-coupled-cavity 


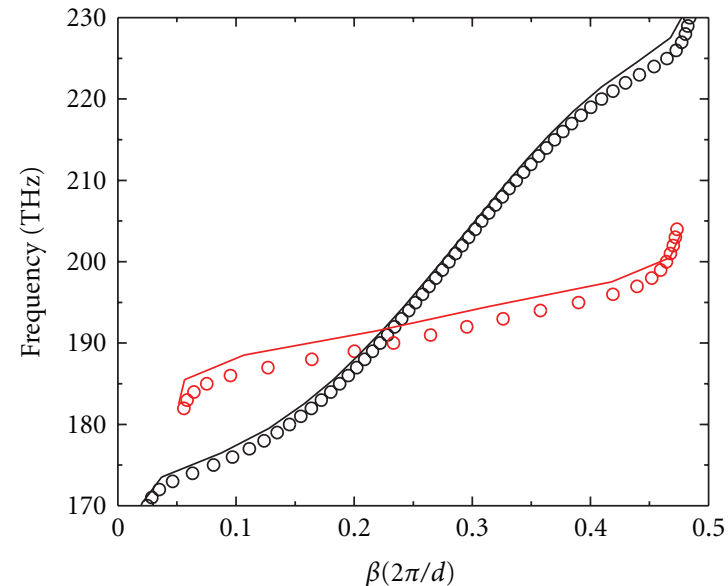

(a)

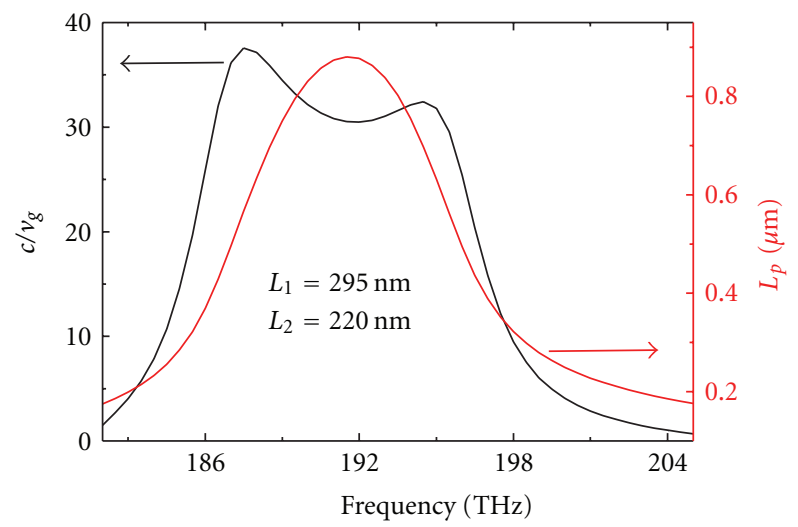

(c)

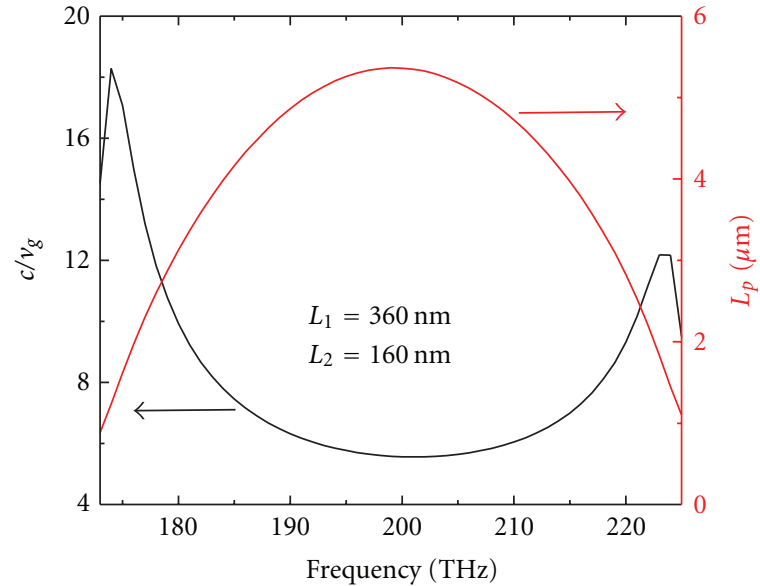

(b)

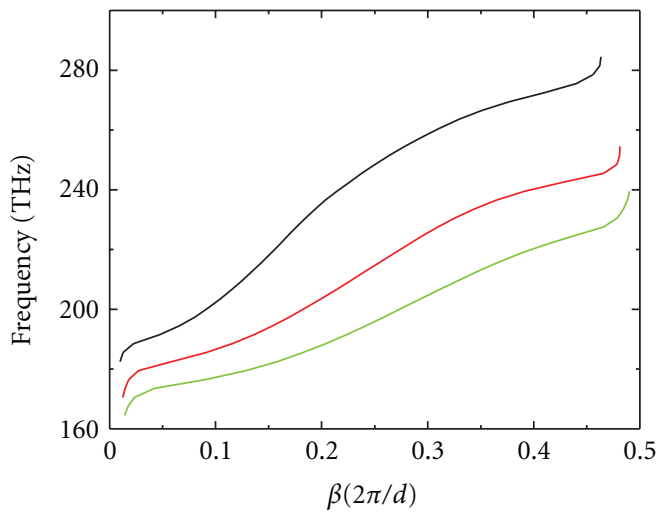

(d)

FIGURE 4: (a) Dispersion relation of the plasmonic waveguide system of Figure 3(a) calculated using FDFD (circles) and scattering matrix theory (solid line). Results are shown for $L_{1}=360 \mathrm{~nm}, L_{2}=160 \mathrm{~nm}$ (black line and circles) and $L_{1}=295 \mathrm{~nm}, L_{2}=220 \mathrm{~nm}($ red line and circles). All other parameters are as in Figure 3(b). In both cases only a portion of the band structure is shown, corresponding to the frequency range of the middle band. ((b)-(c)) Reciprocal of the group velocity $v_{g}$ and propagation length $L_{p}$ for the plasmonic waveguide system of Figure 3(a) as a function of frequency calculated using FDFD. Results are shown for $L_{1}=360 \mathrm{~nm}, L_{2}=160 \mathrm{~nm}$ and $L_{1}=295 \mathrm{~nm}$, $L_{2}=220 \mathrm{~nm}$. All other parameters are as in Figure 3(b). (d) Dispersion relation of the plasmonic waveguide system of Figure 3(a) calculated using FDFD. Results are shown for $d=100 \mathrm{~nm}$ (black line), $d=200 \mathrm{~nm}$ (red line), and $d=300 \mathrm{~nm}$ (green line). All other parameters are as in Figure 3(b). In all cases only a portion of the band structure is shown, corresponding to the frequency range of the middle band.

switch can be calculated using transmission line theory [33] as

$$
T=\left|1+\frac{Z_{2}}{2 Z_{1}} \tanh \left(\gamma_{2} L\right)\right|^{-2}
$$

As seen from (8), the system exhibits a resonance when $\beta_{2} L=(N+1 / 2) \pi$, where $\gamma_{2}=\alpha_{2}+j \beta_{2}$, and $N$ is an integer. We assume that the cavity length $L$ is equal to one of the resonant lengths $L_{N}$ at frequency $\omega_{0}$ and consider the response of the system for frequencies $\omega$ in the vicinity of $\omega_{0}\left(\left|\omega-\omega_{0}\right| / \omega_{0} \ll\right.$ $1)$. In such a case, we find that (8) can be approximated as

$$
T(\omega) \approx \frac{\left(\omega-\omega_{0}\right)^{2}+\left(\omega_{0} / 2 Q_{0}\right)^{2}}{\left(\omega-\omega_{0}\right)^{2}+\left(\left(\omega_{0} / 2 Q_{0}\right)+\left(\omega_{0} / 2 Q_{e}\right)\right)^{2}},
$$

where

$$
Q_{0}=\frac{\omega_{0}}{2 \alpha_{2} v_{g}}, \quad Q_{e}=\frac{Z_{1}}{Z_{2}} \frac{\omega_{0} L_{N}}{v_{g}},
$$

and $v_{g}=\partial \omega / \partial \beta_{2}$. Here $Q_{0}$ is the quality factor associated with the internal loss in the cavity due to the propagation loss of the optical mode and $Q_{e}$ is the quality factor associated with the power escape through the waveguide. We note that (9) can also be directly derived using coupled-mode theory and first-principles calculation of the quality factors $Q_{0}$ and $Q_{e}[64,65]$. We observe that the on-resonance transmission is a function of the ratio $r$ of the quality factors; that is,

$$
T\left(\omega_{0}\right) \approx\left(\frac{r}{r+1}\right)^{2}, \quad r=\frac{Q_{e}}{Q_{0}}=\frac{Z_{1}}{Z_{2}} 2 \alpha_{2} L_{N} .
$$

Since both $\alpha_{2}$ and $Z_{2}$ depend on the imaginary part $\kappa$ of the refractive index in the cavity, the transmission of the system can be controlled by modifying $\kappa$ with an external beam.

In Figure 5(b) we show the transmission spectra of the side-coupled-cavity structure for $w=w_{0}=50 \mathrm{~nm}$ in the absence of optical pumping $(\kappa=0)$ calculated using FDFD. 


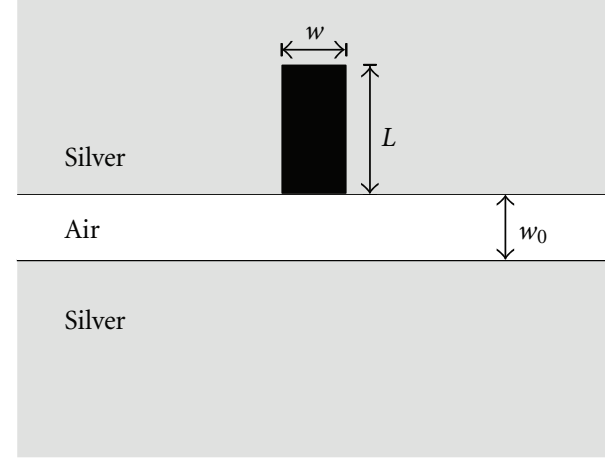

(a)

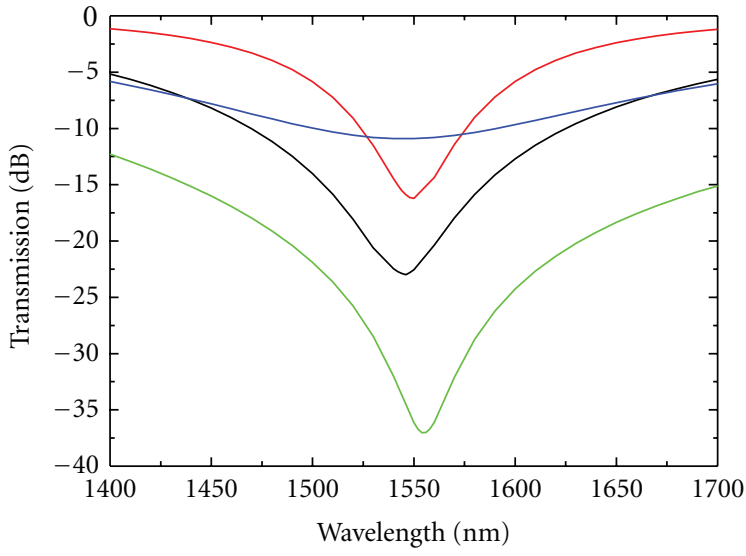

(b)

FIGURE 5: (a) Schematic of a switch consisting of a silver-air-silver MDM plasmonic waveguide side-coupled to a cavity filled with an absorbing material with refractive index $n=2.02+i \kappa$. The imaginary part $\kappa$ of the refractive index can be modified with an external control beam. (b) Transmission spectra of the switch calculated using FDFD. Results are shown for $w=50 \mathrm{~nm}, L=120 \mathrm{~nm}, \kappa=0$ (black curve), $w=50 \mathrm{~nm}, L=405 \mathrm{~nm}, \kappa=0$ (red curve), $w=200 \mathrm{~nm}, L=175 \mathrm{~nm}, \kappa=0$ (green curve), and $w=50 \mathrm{~nm}, L=120 \mathrm{~nm}, \kappa=0.1$ (blue curve). In all cases $w_{0}=50 \mathrm{~nm}$ and $\lambda_{0}=1.55 \mu \mathrm{m}$.

The length of the cavity $L$ is chosen $L=120 \mathrm{~nm}$, so that the system exhibits a resonance at $\lambda_{0}=1.55 \mu \mathrm{m}$. The transmission spectra are characterized by a Lorentzian lineshape, as predicted by (9). We observe that, as $\left|\omega-\omega_{0}\right|$ increases, the transmission increases, and, in the limit $\left|\omega-\omega_{0}\right| \rightarrow \infty$, the transmission approaches $1\left(\lim _{\left|\omega-\omega_{0}\right| \rightarrow \infty} T(\omega)=1\right)$. In other words, if $\omega$ is far from the resonant frequency $\omega_{0}$, the incident waveguide mode is almost completely transmitted. At resonance $\left(\omega=\omega_{0}\right)$, we observe that the transmission is less than $1 \%\left(T\left(\omega_{0}\right) \approx-23 \mathrm{~dB}\right)$. When the material filling the cavity is in its transparent state $(\kappa=0)$, the propagation loss of the optical mode is only associated with the loss in the metal. In that case, the propagation length is in the order of tens of micrometers at near-infrared wavelengths [6], so that $\alpha_{2} L \ll 1$, and therefore $r \ll 1$ and $T \ll 1$ (11). In addition, since $r \ll 1$, the total quality factor, defined as $Q \equiv\left(Q_{0}^{-1}+Q_{e}^{-1}\right)^{-1}$, is $Q \approx Q_{e} \approx 4.4$ (10), and the system response is broad (Figure 5(b)). The low-quality factor in this structure is associated with the low reflectivity at the waveguide-cavity interface due to the small impedance mismatch.

If the stub length $L$ increases to the second resonant length ( $L=405 \mathrm{~nm}$ is chosen as before so that the system exhibits a resonance at $\lambda_{0}=1.55 \mu \mathrm{m}$ ), more energy is stored in the resonant cavity, so that $Q_{e}$ increases (10), and therefore the on-resonance transmission also increases (11). We indeed observe that for $L=405 \mathrm{~nm}$ the transmission is higher than that for $L=120 \mathrm{~nm}$ in the entire frequency range (Figure 5(b)).

If the stub width $w$ increases $(w=200 \mathrm{~nm}$, and $L=$ $175 \mathrm{~nm}$ is chosen as before so that the system exhibits a resonance at $\lambda_{0}=1.55 \mu \mathrm{m}$ ), the propagation length of the optical mode in the cavity increases, leading to higher $Q_{0}$ (10). In addition, the wider $w$ leads to larger power escape through the waveguide and therefore lower $Q_{e}$. Hence the onresonance transmission decreases (11). We indeed observe that for wider $w$ the transmission is lower in the entire frequency range (Figure 5(b)).

In the presence of optical pumping, the material in the stub switches to its absorbing state. The internal loss in the cavity increases, and therefore $Q_{0}$ decreases, resulting in higher on-resonance transmission (11). We indeed observe that for $\kappa=0.1$ the on resonance transmission is significantly larger than that for $\kappa=0$ (Figure 5(b)). Thus, the sidecoupled structure can operate as an absorption switch for MDM plasmonic waveguides, in which the on/off states correspond to the presence/absence of optical pumping.

In Figure 6(a) we show the modulation depth of the switch $T(\kappa=1) / T(\kappa=0)$ as a function of the cavity length $L$ at $\lambda_{0}=1.55 \mu \mathrm{m}$ calculated with FDFD. We observe that the modulation depth exhibits peaks when $L$ is equal to one of the resonant lengths of the stub. This is due to the fact that the transmission in the absence of pumping $T(\kappa=0)$ is minimized on resonance, as described above. We also observe that the maximum modulation depth is obtained when the cavity length $L$ is equal to the first resonant length. As described above, if the stub length $L$ increases to a higherorder resonant length, the quality factor $Q_{e}$ increases and leads to larger on-resonance transmission. This occurs both in the presence and in the absence of pumping; that is, both $T(\kappa=1)$ and $T(\kappa=0)$ increase. In the absence of pumping $(\kappa=0)$, we have $r \ll 1$ for the ratio $r$ of the quality factors, as mentioned above. Thus, based on (11), the on-resonance transmission in the absence of pumping varies roughly quadratically with $r$. On the other hand, in the presence of pumping, $r$ is much larger, and the on-resonance transmission is, therefore, less sensitive to $r$ (11). In other words, if the stub length $L$ increases to a higher-order resonant length, the on-resonance transmission in the absence of pumping $T(\kappa=$ 0 ) increases more than the on-resonance transmission in the presence of pumping $T(\kappa=1)$. Thus, the modulation depth $T(\kappa=1) / T(\kappa=0)$ decreases (Figure 6(a)). In Figure 6(a) 


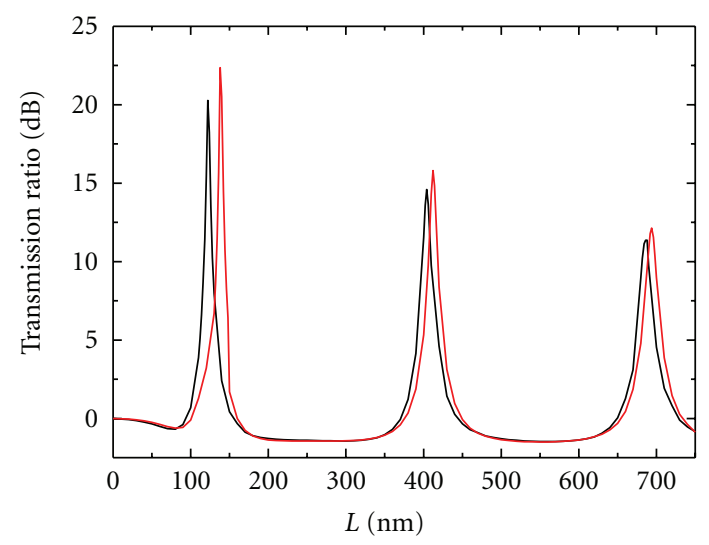

(a)

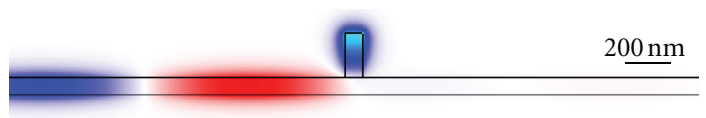

(b)

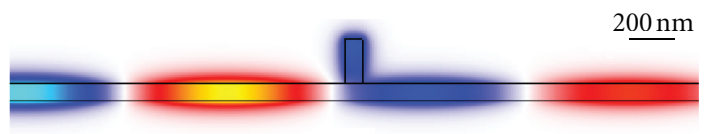

(c)

Figure 6: (a) Modulation depth $T(\kappa=1) / T(\kappa=0)$ of the side-coupled-cavity switch (Figure $5(\mathrm{a})$ ) as a function of the stub length $L$ calculated using FDFD (black curve), and transmission line theory (red curve). Results are shown for $w=50 \mathrm{~nm}$. All other parameters are as in Figure 5(b). (b) Magnetic field profile of the switch for $L=120 \mathrm{~nm}$ in the absence of pumping $(\kappa=0)$. All other parameters are as in (a). (c) Magnetic field profile of the switch in the presence of pumping $(\kappa=1)$. All other parameters are as in $(b)$.

we also show the modulation depth of the switch calculated by transmission line theory (8). We again observe that there is very good agreement between the transmission line theory results and the exact results obtained using FDFD.

In Figures 6(b) and 6(c) we show the magnetic field profile of the side-coupled-cavity switch corresponding to the off and on states, respectively. In the absence of pumping, corresponding to the off state, the incident optical mode is almost completely reflected. In contrast, in the presence of pumping, corresponding to the on state, the transmission increases by more than two orders of magnitude leading to a large modulation depth.

In Figure 7 we show the maximum modulation depth of the side-coupled-cavity switch as a function of the stub width $w$. As mentioned above, for a given $w$ the maximum modulation depth is achieved when the stub length $L$ is equal to the first resonant length. We observe that for $w<$ $300 \mathrm{~nm}$ the maximum modulation depth increases with $w$. As described above, larger $w$ leads to both higher $Q_{0}$ and lower $Q_{e}$ for the resonator. Thus, the quality factors' ratio $r$ decreases, and the on-resonance transmission decreases (11). This occurs both in the presence and in the absence of pumping; that is, both $T(\kappa=1)$ and $T(\kappa=0)$ decrease. However, as mentioned above, in the absence of

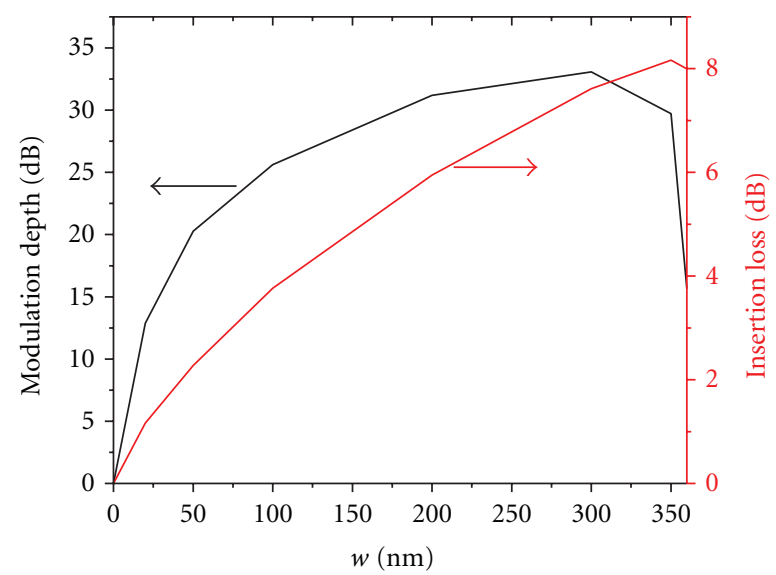

FIgUre 7: Modulation depth $T(\kappa=1) / T(\kappa=0)$ (black curve) and insertion loss (red curve) of the side-coupled-cavity switch (Figure 5(a)) as a function of the stub width $w$. For each stub width $w$, the stub length $L$ is equal to the first resonant length of the cavity. All other parameters are as in Figure 5(b).

pumping $(\kappa=0)$ the on-resonance transmission is more sensitive to $r$. Thus, as the stub width $w$ increases, the onresonance transmission in the absence of pumping $T(\kappa=0)$ decreases more than the on-resonance transmission in the presence of pumping $T(\kappa=1)$. Thus, the modulation depth $T(\kappa=1) / T(\kappa=0)$ increases with $w$ (Figure 7$)$. We also observe that the modulation depth is maximized for $w \approx$ $300 \mathrm{~nm}$. In other words, for a given pumping intensity there is a maximum achievable modulation depth for the sidecoupled-cavity structure. We found that the decrease with $w$ of the modulation depth for $w>300 \mathrm{~nm}$ is associated with the excitation of higher-order modes in the resonator, which occurs when $w$ becomes comparable to the wavelength. In this regime, the transmission line model breaks down, and the system properties are no longer accurately described by (8)-(11).

In Figure 7 we also show the insertion loss of the sidecoupled-cavity switch, defined as $-10 \log _{10}(T(\kappa=1))$, as a function of the stub width $w$. As described above, for $w<300 \mathrm{~nm}$ the on-resonance transmission in the presence of pumping $T(\kappa=1)$ decreases with $w$, and the insertion loss therefore increases (Figure 7). For $w>300 \mathrm{~nm}$, the insertion loss decreases with $w$ (Figure 7$)$, due to the excitation of higher-order modes in the resonator, as also described above. We observe that for the side-coupled-cavity switch there is a tradeoff between modulation depth and insertion loss, as the geometrical parameters of the stub are varied. Similar tradeoffs are observed in electroabsorption modulators [66].

In Figure 8, we show the modulation depth $T(\kappa) / T(\kappa=$ 0 ) of the side-coupled-cavity switch as a function of the imaginary part $\kappa$ of the refractive index. As expected, the modulation depth increases with $\kappa$. We also observe that even for a relatively small variation in the absorption coefficient of the material filling the cavity $(\kappa=0.01)$, we can achieve a modulation depth of $\sim 60 \%(\sim 4 \mathrm{~dB})$. We note that such modulation depths have been demonstrated experimentally 


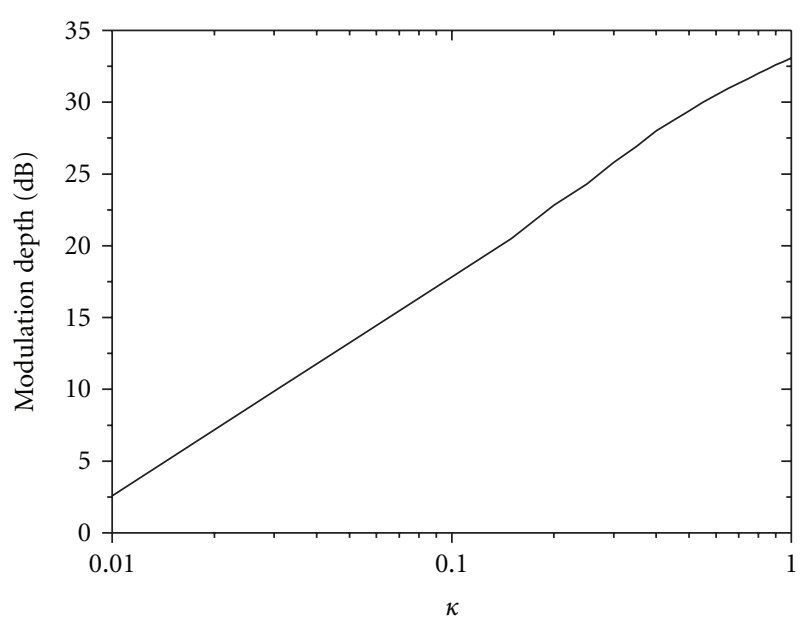

Figure 8: Modulation depth $T(\kappa) / T(\kappa=0)$ of the side-coupledcavity switch (Figure 5(a)) as a function of the imaginary part $\kappa$ of the refractive index. Results are shown for $w=300 \mathrm{~nm}, L=210 \mathrm{~nm}$. All other parameters are as in Figure 5(b).

in other plasmonic absorption switches [23, 63]. For a modulation depth of $99 \%$, the required variation is $\kappa=0.15$.

\section{Conclusions}

In summary, in this paper we provided a review of some of our recent research activities on plasmonic devices based on MDM stub resonators for manipulating light at the nanoscale. We first briefly reviewed the methods used for the simulation and analysis of such devices. We then introduced slow-light subwavelength plasmonic waveguides based on plasmonic analogues of periodically loaded transmission lines and electromagnetically induced transparency. In both cases, the structures consist of a MDM waveguide sidecoupled to periodic arrays of MDM stub resonators. We finally introduced switches consisting of a MDM plasmonic waveguide side-coupled to a MDM stub resonator filled with an active material. As final remarks, we note that plasmonic devices based on MDM stub resonators have also been proposed as compact filters, reflectors, and impedance matching elements for MDM plasmonic waveguides [10, 6774].

\section{References}

[1] W. L. Barnes, A. Dereux, and T. W. Ebbesen, "Surface plasmon subwavelength optics," Nature, vol. 424, no. 6950, pp. 824830, 2003.

[2] E. Ozbay, "Plasmonics: merging photonics and electronics at nanoscale dimensions," Science, vol. 311, no. 5758, pp. 189193,2006

[3] J. R. Krenn, B. Lamprecht, H. Ditlbacher et al., "Non-diffraction-limited light transport by gold nanowires," Europhysics Letters, vol. 60, no. 5, pp. 663-669, 2002.

[4] S. A. Maier, P. G. Kik, H. A. Atwater et al., "Local detection of electromagnetic energy transport below the diffraction limit in metal nanoparticle plasmon waveguides," Nature Materials, vol. 2, no. 4, pp. 229-232, 2003.
[5] S. I. Bozhevolnyi, V. S. Volkov, E. Devaux, J. Y. Laluet, and T. W. Ebbesen, "Channel plasmon subwavelength waveguide components including interferometers and ring resonators," Nature, vol. 440, no. 7083, pp. 508-511, 2006.

[6] R. Zia, M. D. Selker, P. B. Catrysse, and M. L. Brongersma, "Geometries and materials for subwavelength surface plasmon modes," Journal of the Optical Society of America A, vol. 21, no. 12, pp. 2442-2446, 2004.

[7] G. Veronis and S. Fan, "Bends and splitters in metal-dielectricmetal subwavelength plasmonic waveguides," Applied Physics Letters, vol. 87, Article ID 131102, 3 pages, 2005.

[8] A. Hosseini and Y. Massoud, "Nanoscale surface plasmon based resonator using rectangular geometry," Applied Physics Letters, vol. 90, no. 18, Article ID 181102, 3 pages, 2007.

[9] Y. Matsuzaki, T. Okamoto, M. Haraguchi, M. Fukui, and M. Nakagaki, "Characteristics of gap plasmon waveguide with stub structures," Optics Express, vol. 16, no. 21, pp. 1631416325, 2008.

[10] X. S. Lin and X. G. Huang, "Tooth-shaped plasmonic waveguide filters with nanometeric sizes," Optics Letters, vol. 33, no. 23, pp. 2874-2876, 2008.

[11] E. N. Economou, "Surface plasmons in thin films," Physical Review, vol. 182, no. 2, pp. 539-554, 1969.

[12] J. A. Dionne, L. A. Sweatlock, H. A. Atwater, and A. Polman, "Planar metal plasmon waveguides: frequency-dependent dispersion, propagation, localization, and loss beyond the free electron model," Physical Review B, vol. 72, Article ID 075405, 11 pages, 2005.

[13] J. A. Dionne, L. A. Sweatlock, H. A. Atwater, and A. Polman, "Plasmon slot waveguides: towards chip-scale propagation with subwavelength-scale localization," Physical Review B, vol. 73, Article ID 035407, 9 pages, 2006.

[14] S. E. Kocabas, G. Veronis, D. A. B. Miller, and S. Fan, "Modal analysis and coupling in metal-insulator-metal waveguides," Physical Review B, vol. 79, Article ID 035120, 17 pages, 2009.

[15] J. A. Dionne, H. J. Lezec, and H. A. Atwater, "Highly confined photon transport in subwavelength metallic slot waveguides," Nano Letters, vol. 6, no. 9, pp. 1928-1932, 2006.

[16] H. J. Lezec, J. A. Dionne, and H. A. Atwater, "Negative refraction at visible frequencies," Science, vol. 316, no. 5823, pp. 430-432, 2007.

[17] E. Verhagen, J. A. Dionne, L. Kuipers, H. A. Atwater, and A. Polman, "Near-field visualization of strongly confined surface plasmon polaritons in metal-insulator-metal waveguides," Nano Letters, vol. 8, no. 9, pp. 2925-2929, 2008.

[18] Z. Han, A. Y. Elezzabi, and Van, "Experimental realization of subwavelength plasmonic slot waveguides on a silicon platform," Optics Letters, vol. 35, no. 4, pp. 502-504, 2010.

[19] Z. Han, A. Y. Elezzabi, and V. Van, "Wideband Y-splitter and aperture-assisted coupler based on sub-diffraction confined plasmonic slot waveguides," Applied Physics Letters, vol. 96, no. 13, Article ID 131106, 3 pages, 2010.

[20] S. Zhu, T. Y. Liow, G. Q. Lo, and D. L. Kwong, "Fully complementary metal-oxide-semiconductor compatible nanoplasmonic slot waveguides for silicon electronic photonic integrated circuits," Applied Physics Letters, vol. 98, no. 2, Article ID 021107, 3 pages, 2011.

[21] J. Wen, P. Banzer, A. Kriesch, D. Ploss, B. Schmauss, and U. Peschel, "Experimental cross-polarization detection of coupling far-field light to highly confined plasmonic gap modes via nanoantennas," Applied Physics Letters, vol. 98, no. 10, Article ID 101109, 2011. 
[22] D. Pacifici, H. J. Lezec, and H. A. Atwater, "All-optical modulation by plasmonic excitation of CdSe quantum dots," Nature Photonics, vol. 1, no. 7, pp. 402-406, 2007.

[23] R. A. Pala, K. T. Shimizu, N. A. Melosh, and M. L. Brongersma, "A nonvolatile plasmonic switch employing photochromic molecules," Nano Letters, vol. 8, no. 5, pp. 1506-1510, 2008.

[24] C. Min and G. Veronis, "Absorption switches in metaldielectric-metal plasmonic waveguides," Optics Express, vol. 17, no. 13, pp. 10757-10766, 2009.

[25] L. Yang, C. Min, and G. Veronis, "Guided subwavelength slowlight mode supported by a plasmonic waveguide system," Optics Letters, vol. 35, no. 24, pp. 4184-4186, 2010.

[26] Y. Huang, C. Min, and G. Veronis, "Subwavelength slow-light waveguides based on a plasmonic analogue of electromagnetically induced transparency," Applied Physics Letters, vol. 99, no. 14, Article ID 143117, 2011.

[27] S.-D. Wu and E. N. Glytsis, "Finite-number-of-periods holographic gratings with finite-width incident beams: analysis using the finite-difference frequency-domain method," Journal of the Optical Society of America A, vol. 19, no. 10, pp. 2018-2029, 2002.

[28] G. Veronis, R. W. Dutton, and S. Fan, "Method for sensitivity analysis of photonic crystal devices," Optics Letters, vol. 29, no. 19, pp. 2288-2290, 2004.

[29] E. D. Palik, Handbook of Optical Constants of Solids, Academic, Orlando, Fla, USA, 1985.

[30] J. Jin, The Finite Element Method in Electromagnetics, Wiley, New York, NY, USA, 2002.

[31] A. Taflove and S. C. Hagness, Computational Electrodynamics, Artech House, Boston, Mass, USA, 3rd edition, 2005.

[32] M. N. O. Sadiku, Numerical Techniques in Electromagnetics, CRC Press, Boca Raton, Fla, USA, 2001.

[33] D. M. Pozar, Microwave Engineering, Wiley, New York, NY, USA, 1998.

[34] S. Ramo, J. R. Whinnery, and T. Van, Duzer, Fields and Waves in Communication Electronics, Wiley, New York, NY, USA, 1994.

[35] S. E. Kocabas, G. Veronis, D. A. B. Miller, and S. Fan, "Transmission line and equivalent circuit models for plasmonic waveguide components," IEEE Journal on Selected Topics in Quantum Electronics, vol. 14, no. 6, Article ID 4559744, pp. 1462-1472, 2008.

[36] R. D. Kekatpure, E. S. Barnard, W. Cai, and M. L. Brongersma, "Phase-coupled plasmon-induced transparency," Applied Physics Letters, vol. 104, Article ID 243902, 4 pages, 2010.

[37] Z. Han and S. I. Bozhevolnyi, "Plasmon-induced transparency with detuned ultracompact Fabry-Perot resonators in integrated plasmonic devices," Optics Express, vol. 19, no. 4, pp. 3251-3257, 2011.

[38] D. Pacifici, H. J. Lezec, H. A. Atwater, and J. Weiner, "Quantitative determination of optical transmission through subwavelength slit arrays in Ag films: role of surface wave interference and local coupling between adjacent slits," Physical Review B, vol. 77, Article ID 115411, 2008.

[39] D. Pacifici, H. J. Lezec, L. A. Sweatlock, R. J. Walters, and H. A. Atwater, "Universal optical transmission features in periodic and quasiperiodic hole arrays," Optics Express, vol. 16, no. 12, pp. 9222-9238, 2008.

[40] A. Karalis, E. Lidorikis, M. Ibanescu, J. Joannopoulos, and M. Soljacic, "Surface-plasmon-assisted guiding of broadband slow and subwavelength light in air," Physical Review Letters, vol. 95, Article ID 063901, 2005.
[41] M. Sandtke and L. Kuipers, "Slow guided surface plasmons at telecom frequencies," Nature Photonics, vol. 1, no. 10, pp. 573576, 2007.

[42] Z. Kang, W. Lin, and G. P. Wang, "Dual-channel broadband slow surface plasmon polaritons in metal gap waveguide superlattices," Journal of the Optical Society of America B, vol. 26, no. 10, pp. 1944-1948, 2009.

[43] Q. Gan, Y. J. Ding, and F. J. Bartoli, "Rainbow' trapping and releasing at telecommunication wavelengths," Physical Review Letters, vol. 102, Article ID 056801, 4 pages, 2009.

[44] A. Kocabas, S. S. Senlik, and A. Aydinli, "Slowing down surface plasmons on a Moiré surface," Physical Review Letters, vol. 102, Article ID 063901, 4 pages, 2009.

[45] E. Feigenbaum and M. Orenstein, "Backward propagating slow light in inverted plasmonic taper," Optics Express, vol. 17, no. 4, pp. 2465-2469, 2009.

[46] S. A. Maier, Plasmonics: Fundamentals and Applications, Springer, New York, NY, USA, 2007.

[47] P. Tassin, L. Zhang, T. Koschny, E. N. Economou, and C. M. Soukoulis, "Low-loss metamaterials based on classical electromagnetically induced transparency," Physical Review Letters, vol. 102, no. 5, Article ID 053901, 2009.

[48] S. Fan, M. F. Yanik, Z. Wang, S. Sandhu, and M. L. Povinelli, "Advances in theory of photonic crystals," Journal of Lightwave Technology, vol. 24, no. 12, pp. 4493-4501, 2006.

[49] S. Zhang, D. A. Genov, Y. Wang, M. Liu, and X. Zhang, "Plasmon-induced transparency in metamaterials," Physical Review Letters, vol. 101, no. 4, Article ID 047401, 2008.

[50] N. Papasimakis, V. A. Fedotov, and N. I. Zheludev, "Metamaterial analog of electromagnetically induced transparency," Physical Review Letters, vol. 101, Article ID 253903, 4 pages, 2008.

[51] N. Liu, L. Langguth, T. Weiss et al., "Plasmonic analogue of electromagnetically induced transparency at the Drude damping limit," Nature Materials, vol. 8, no. 9, pp. 758-762, 2009.

[52] M. F. Yanik, W. Suh, Z. Wang, and S. Fan, "Stopping light in a waveguide with an all-optical analog of electromagnetically induced transparency," Physical Review Letters, vol. 93, Article ID 233903, 1 pages, 2004.

[53] Q. Xu, S. Sandhu, M. Povinelli, J. Shakya, S. Fan, and M. Lipson, "Experimental realization of an on-chip all-optical analogue to electromagnetically induced transparency," Physical Review Letters, vol. 96, no. 12, Article ID 123901, 4 pages, 2006.

[54] A. V. Krasavin and N. I. Zheludev, "Active plasmonics: controlling signals in $\mathrm{Au} / \mathrm{Ga}$ waveguide using nanoscale structural transformations," Applied Physics Letters, vol. 84, no. 8, pp. 1416-1418, 2004.

[55] T. Nikolajsen, K. Leosson, and S. I. Bozhevolnyi, "Surface plasmon polariton based modulators and switches operating at telecom wavelengths," Applied Physics Letters, vol. 85, no. 24, pp. 5833-5835, 2004.

[56] A. L. Lereu, A. Passian, J. P. Goudonnet, T. Thundat, and T. L. Ferrell, "Optical modulation processes in thin films based on thermal effects of surface plasmons," Applied Physics Letters, vol. 86, no. 15, Article ID 154101, pp. 1-3, 2005.

[57] K. F. MacDonald, Z. L. Sámson, M. I. Stockman, and N. I. Zheludev, "Ultrafast active plasmonics," Nature Photonics, vol. 3, no. 1, pp. 55-58, 2009.

[58] G. A. Wurtz and A. V. Zayats, "Nonlinear surface plasmon polaritonic crystals," Laser and Photonics Reviews, vol. 2, no. 3, pp. 125-135, 2008. 
[59] C. Min, P. Wang, C. Chen et al., "All-optical switching in subwavelength metallic grating structure containing nonlinear optical materials," Optics Letters, vol. 33, no. 8, pp. 869-871, 2008.

[60] M. J. Dicken, L. A. Sweatlock, D. Pacifici, H. J. Lezec, K. Bhattacharya, and H. A. Atwater, "Electrooptic modulation in thin film barium titanate plasmonic interferometers," Nano Letters, vol. 8, no. 11, pp. 4048-4052, 2008.

[61] W. Dickson, G. A. Wurtz, P. R. Evans, R. J. Pollard, and A. V. Zayats, "Electronically controlled surface plasmon dispersion and optical transmission through metallic hole arrays using liquid crystal," Nano Letters, vol. 8, no. 1, pp. 281-286, 2008.

[62] Z. Yu, G. Veronis, S. Fan, and M. L. Brongersma, "Gaininduced switching in metal-dielectric-metal plasmonic waveguides," Applied Physics Letters, vol. 92, no. 4, Article ID 041117, 3 pages, 2008.

[63] D. Pacifici, H. J. Lezec, L. A. Sweatlock, C. D. Ruiter, V. Ferry, and H. A. Atwater, Plasmonic Nanoguides and Circuits, World Scientific, River Edge, NJ, USA, 2009.

[64] H. A. Haus and Y. Lai, "Narrow-band distributed feedback reflector design," Journal of Lightwave Technology, vol. 9, no. 6, pp. 754-760, 1991.

[65] H. A. Haus and Y. Lai, "Theory of cascaded quarter wave shifted distributed feedback resonators," IEEE Journal of Quantum Electronics, vol. 28, no. 1, pp. 205-213, 1992.

[66] J. Liu, M. Beals, A. Pomerene et al., "Waveguide-integrated, ultralow-energy GeSi electro-absorption modulators," Nature Photonics, vol. 2, no. 7, pp. 433-437, 2008.

[67] A. Pannipitiya, I. D. Rukhlenko, M. Premaratne, H. T. Hattori, and G. P. Agrawal, "Improved transmission model for metaldielectric-metal plasmonic waveguides with stub structure," Optics Express, vol. 18, no. 6, pp. 6191-6204, 2010.

[68] Z. Zhong, Y. Xu, S. Lan, Q. Dai, and L. Wu, "Sharp and asymmetric transmission response in metal-dielectric-metal plasmonic waveguides containing Kerr nonlinear media," Optics Express, vol. 18, no. 1, pp. 79-68, 2010.

[69] J. Liu, G. Fang, H. Zhao, Y. Zhang, and S. Liu, "Surface plasmon reflector based on serial stub structure," Optics Express, vol. 17, no. 22, pp. 20134-20139, 2009.

[70] F. Hu, H. Yi, and Z. Zhou, "Wavelength demultiplexing structure based on arrayed plasmonic slot cavities," Optics Letters, vol. 36, no. 8, pp. 1500-1502, 2011.

[71] Y. Xu, A. E. Miroshnichenko, S. Lan, Q. Guo, and L. J. Wu, "Impedance matching induce high transmission and flat responseb band-pass plasmonic waveguides," Plasmonics, vol. 6, no. 2, pp. 337-343, 2011.

[72] Q. Zhang, X. Huang, X. Lin, J. Tao, and X. Jin, "A subwavelength coupler-type MIM optical filter," Optics Express, vol. 17, no. 9, pp. 7549-7555, 2009.

[73] J. Tao, Q. J. Wang, and X. G. Huang, "All-optical plasmonic switches based on coupled nano-disk cavity structures containing nonlinear material," Plasmonics, vol. 6, no. 4, pp. 753$759,2011$.

[74] S. R. Mirnaziry, A. Setayesh, and M. S. Abrishamian, "Design and analysis of plasmonic filters based on stubs," Journal of the Optical Society of America B, vol. 28, no. 5, pp. 1300-1307, 2011. 

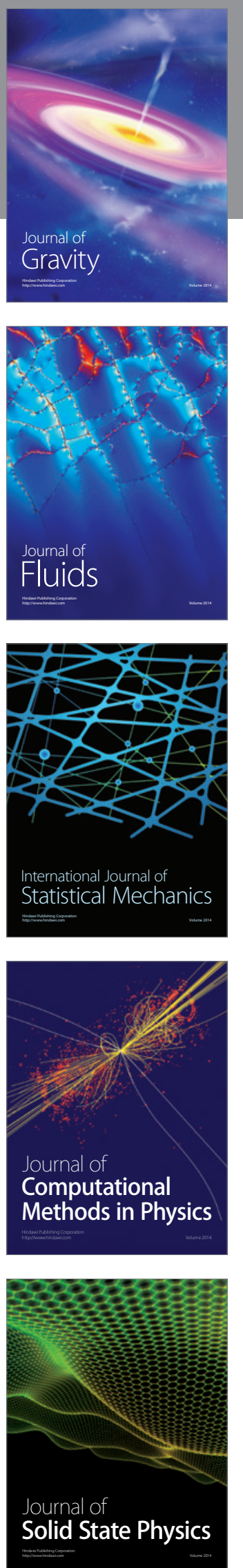

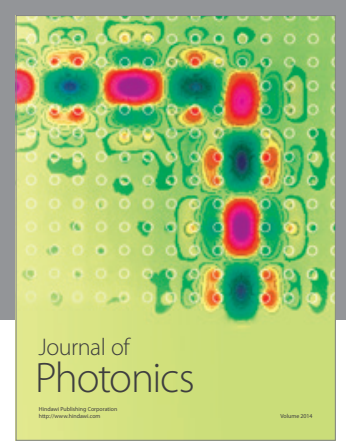

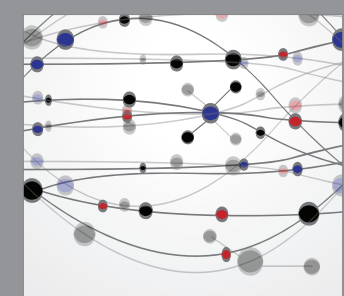

The Scientific World Journal
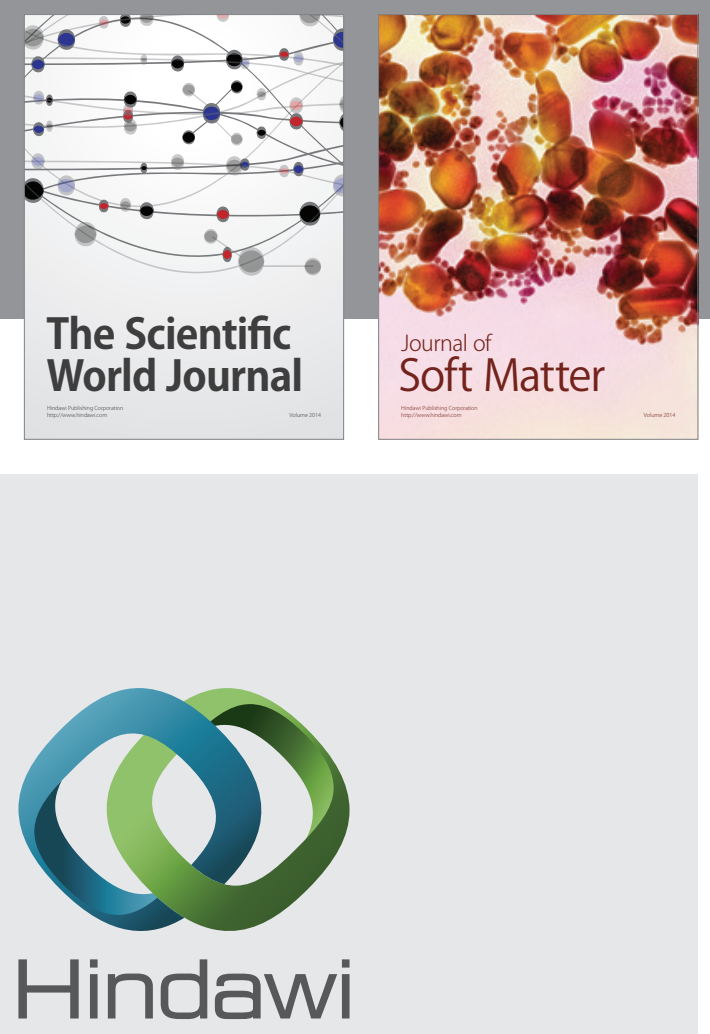

Submit your manuscripts at

http://www.hindawi.com
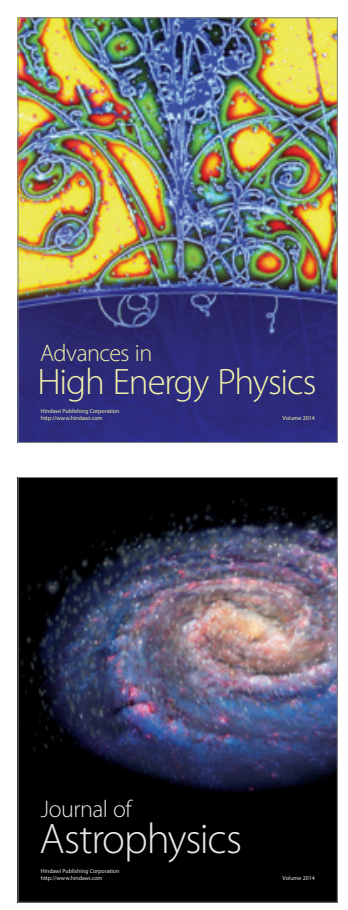
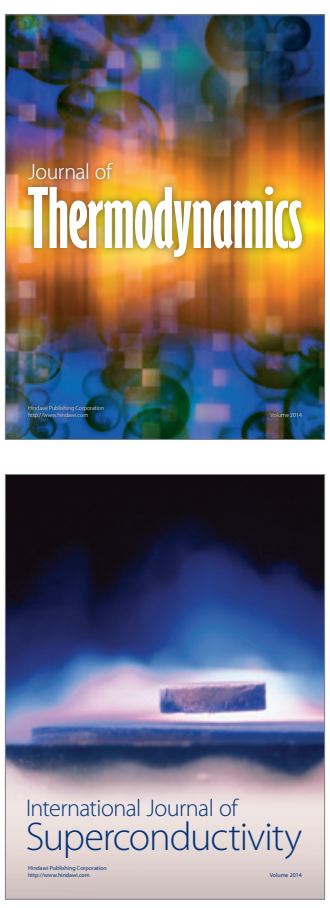
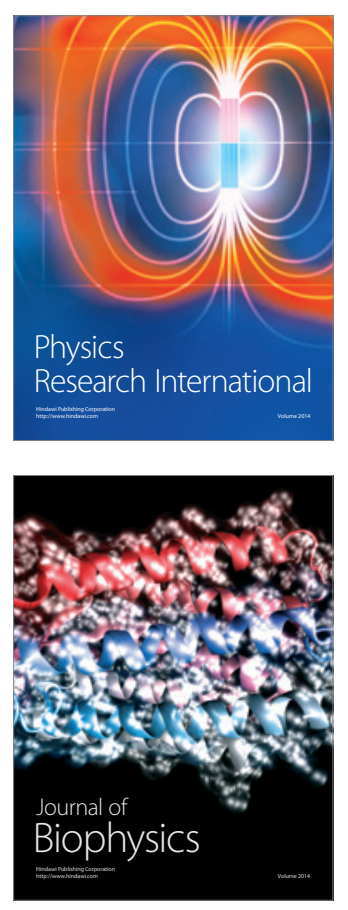
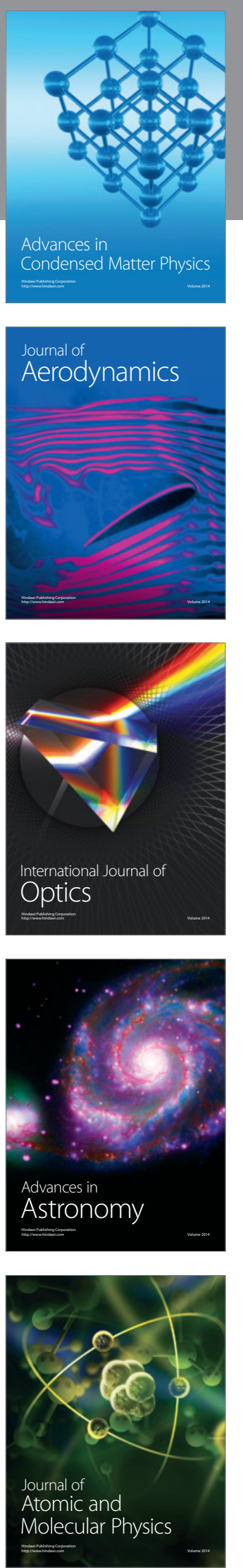